\title{
Pregnant Women's Knowledge and Beliefs about the Safety and Outcomes of Delivery at Various Gestational Ages
}

\author{
Melody A. Baldwin, MD, MPH ${ }^{1}$ Geeta K. Swamy, MD² Sarahn M. Wheeler, MD² \\ ${ }^{1}$ Department of Obstetrics and Gynecology, Duke University School \\ of Medicine, Durham, North Carolina \\ 2 Division of Maternal-Fetal Medicine, Department of Obstetrics and \\ Gynecology, Duke University School of Medicine, Durham, \\ North Carolina \\ Address for correspondence Sarahn Wheeler, MD, Division of \\ Maternal-Fetal Medicine, Department of Obstetrics and Gynecology, \\ Duke University School of Medicine, 2608 Erwin Road Suite \#210, \\ Durham, NC 27710 (e-mail: sarahn.wheeler@duke.edu).
}

Am J Perinatol Rep 2018;8:e7-e12.

\begin{abstract}
Keywords

- gestational age

- patient's perception

- preterm birth

- preterm delivery

- neonatal risk

Objectives Despite the morbidity associated with late preterm and early-term births, there is limited data on pregnant women's perception of neonatal risk based on gestational age (GA). Therefore, our objective was to determine pregnant women's perception of neonatal risks at varying GAs.

Method Through an anonymous 24-question survey, pregnant women were asked to designate the GA at delivery that is desirable, safe, and defined as full term. Responses were compared based on race, history of preterm birth, and medical comorbidities.

Results Among the 233 survey respondents, the majority (62.9\%) desired delivery at 36 to 39 weeks' gestation. Black women were more likely to desire delivery at 28 to 35 weeks compared with other racial/ethnic groups $(p=0.005)$. Women with a history of preterm birth or medical complications were less likely to desire delivery at 40 weeks. More than $40 \%$ of respondents thought delivery at 8 months of pregnancy was safe and $40.3 \%$ responded that 37 weeks' gestation is considered term.

Conclusion Misconceptions surrounding the definition of a term pregnancy are pervasive and vary by race, obstetric history, and medical comorbidities. Our findings highlight the need for patient education about appropriate gestational length, especially in minority and high-risk populations.
\end{abstract}

Preterm birth (prior to 37 weeks' gestation) is a leading cause of neonatal morbidity and mortality. In recent years, there is an increasing understanding of the perinatal and long-term health complications related to late preterm (3436 weeks' gestation) and early-term (37-38 weeks' gestation) births. ${ }^{1-3}$ In 2013, the American Congress of Obstetricians and Gynecologists (ACOG) and the National Institutes of Child Health and Human Development (NICHD) defined early term (37-38 $8^{6 / 7}$ weeks), full term (39-40 6 weeks), late term (41-41 ${ }^{6 / 7}$ weeks), and postterm (42 weeks and beyond). ${ }^{4,5}$ Studies show that women's perception of neo-

received

September 19, 2017 accepted after revision December 9, 2017
DOI https://doi.org/

10.1055/s-0038-1624561. ISSN 2157-6998. natal risk based on gestational age (GA) at delivery vary widely. In a survey of postpartum women with uncomplicated pregnancies, Goldenberg et al noted that $24 \%$ of women believed that full-term gestation occurred at 34 to 36 weeks and nearly half believed that it is safe to deliver before 37 weeks. $^{6}$ In another survey of the general population, Massett et al found that most people believe prematurity is a threat to neonates and that very preterm birth poses a significant risk to neonates; however, only one-third of women and one quarter of men viewed preterm birth as a serious problem in the United States. ${ }^{7}$
Copyright $\odot 2018$ by Thieme Medical Publishers, Inc., 333 Seventh Avenue, New York, NY 10001, USA. Tel: +1(212) 584-4662.
License terms

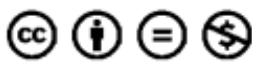


There is very limited data on pregnant patient's perception of neonatal risk based on GA at delivery. Further, it is unclear how factors that influence preterm birth risk such as race, obstetrical history, and medical comorbidities influence pregnant women's perceptions of neonatal risk based on GA at delivery. Our objective was to investigate patient's attitudes and beliefs regarding the desirability, safety, and risk of adverse neonatal outcomes at various GAs. We further sought to determine if these beliefs are correlated with race, obstetrical history, or medical comorbidities during pregnancy.

\section{Methods}

We conducted an anonymous, written survey of pregnant women in three obstetric clinics affiliated with Duke University Health System. Two of the clinics were private lowrisk general obstetrics practices and the third was a mixed public/private high-risk obstetrics clinic. Prior to survey administration, the study was declared exempt from further review by the Duke University Health System Institutional Review Board. Surveys were administered over a 6-week time period. Clinic nursing staff asked all pregnant, Englishliterate women to complete the 24-question anonymous survey during routine prenatal care visits after appointment check-in. The patient's current trimester was marked on the survey cover sheet by clinic staff. Completed surveys were returned to nursing or administrative staff. Exclusion criteria included non-English literacy and prior completion of the survey during their current pregnancy.

Participants were asked to record sociodemographic information including age, race/ethnicity, marital status, educational attainment, and zip code, as well as relevant medical history, including tobacco use and obstetric history. Participants were then asked to report on their experience with the following pregnancy complications either in the current or prior pregnancy: preterm labor, hypertension, diabetes (gestational or preexisting), cervical incompetence, fetal growth restriction, or fetal anomalies. The remainder of the survey assessed pregnant women's perceptions of the GA at which delivery was desirable and safe, as well as what GA (in weeks) they considered full term. The desired GA at delivery and the earliest GA at which delivery is perceived as safe were both asked in separate questions with responses in gestational weeks and months. Beliefs about the likelihood of various birth outcomes (neonatal intensive care unit [NICU] admission, breastfeeding, long-term developmental sequelae, and timing of infant discharge relative to maternal discharge) in the setting of preterm birth were assessed using a Likert scale ranging from very likely to very unlikely. Finally, the survey asked whether or not patients had discussed prematurity with their provider, whether they themselves were born prematurely, and whether they had family or friends that experienced preterm birth with or without NICU admission.

Statistical analysis was performed using JMP, Version 10 (SAS Institute Inc., Cary, NC). Descriptive statistics were calculated for all sociodemographic characteristics, medical history, and survey measures. These include measures of central tendency for continuous variables and frequencies for categorical variables. Univariate analysis was performed using chi-square tests, with a $p$-value of $<0.05$ considered statistically significant.

\section{Results}

Two hundred thirty-three pregnant women completed the survey, providing a maximum $6.4 \%$ margin of error for the estimated proportions. Respondents were diverse in age, race/ethnicity, insurance status, educational attainment, and marital status (-Table $\mathbf{1}$ ). Most patients were age 20 to $29(45.7 \%)$ or 30 to 39 years $(44.4 \%)$. A relatively similar proportion of women within the study were white (46.4\%) and black (41.6\%) race. Over half of women surveyed had Medicaid, while the remainder had private insurance coverage. Nearly equal proportions of patients had obtained a high school, college, or graduate degree. Fifty-seven percent of women surveyed were married.

Nine percent of respondents were active smokers and 11\% were themselves born prematurely ( - Table 2 ). The majority of patients were in the third trimester of pregnancy (39.9\%);

Table 1 Participants' sociodemographic characteristics $(n=233)$

\begin{tabular}{|c|c|}
\hline Characteristics & Total, $n$ (\%) \\
\hline \multicolumn{2}{|l|}{ Age } \\
\hline $15-19$ & $14(6.0)$ \\
\hline $20-29$ & $106(45.7)$ \\
\hline $30-39$ & $103(44.4)$ \\
\hline $40-46$ & $8(3.5)$ \\
\hline \multicolumn{2}{|l|}{ Race/ethnicity } \\
\hline Asian & $12(5.2)$ \\
\hline Black & $97(41.6)$ \\
\hline Hispanic & $9(3.9)$ \\
\hline Other & $7(3.0)$ \\
\hline White & $108(46.4)$ \\
\hline \multicolumn{2}{|l|}{ Insurance status } \\
\hline Medicaid & $119(52)$ \\
\hline Private & $108(47.2)$ \\
\hline Self-pay & $2(0.8)$ \\
\hline \multicolumn{2}{|l|}{ Education } \\
\hline Less than high school & $10(4.3)$ \\
\hline High school & $53(22.7)$ \\
\hline Some college & $57(24.5)$ \\
\hline 2 y college degree & $14(6.0)$ \\
\hline 4 y college degree & $43(18.5)$ \\
\hline Graduate degree & $56(24.0)$ \\
\hline Married & $133(57.1)$ \\
\hline
\end{tabular}


Table 2 Medical and obstetric history $(n=233)$

\begin{tabular}{|c|c|}
\hline Medical history & Total, $n(\%)$ \\
\hline \multicolumn{2}{|l|}{ Tobacco use } \\
\hline Current smoker & $21(9.1)$ \\
\hline Ever smoked & $83(35.6)$ \\
\hline Maternal preterm birth & $26(11.2)$ \\
\hline \multicolumn{2}{|l|}{ Current trimester } \\
\hline First & $25(10.7)$ \\
\hline Second & $63(27.0)$ \\
\hline Third & $93(39.9)$ \\
\hline Unknown & $52(22.3)$ \\
\hline Nulliparous & $99(42.5)$ \\
\hline History of prior pregnancy loss & $73(31.3)$ \\
\hline \multicolumn{2}{|l|}{ Prior pregnancy complications } \\
\hline Preterm birth & $41(17.6)$ \\
\hline Hypertensive disorder & $36(15.5)$ \\
\hline Diabetes (gestational or preexisting) & $23(9.9)$ \\
\hline Cervical incompetence & $21(9.0)$ \\
\hline Fetal anomalies & $4(1.7)$ \\
\hline Low birth weight & $19(8.2)$ \\
\hline \multicolumn{2}{|l|}{ Current pregnancy complications } \\
\hline Preterm labor & $8(3.4)$ \\
\hline Hypertensive disorder & $30(12.9)$ \\
\hline Diabetes (gestational or preexisting) & $32(13.7)$ \\
\hline Cervical incompetence & $15(6.5)$ \\
\hline Fetal anomalies & $4(1.7)$ \\
\hline Fetal growth restriction & $13(5.6)$ \\
\hline
\end{tabular}

however, current trimester was not reported for $22 \%$ of respondents. Only $42.5 \%$ were nulliparous and $31.3 \%$ reported a prior pregnancy loss. Overall, $37 \%$ of women reported a prior pregnancy complication, the most common of which were preterm birth and hypertensive disorders.
Similarly, one-third reported a complication in their current pregnancy, the most common of which were diabetes and hypertensive disorders.

\section{Desired Gestational Age at Delivery}

Participants were asked "How far along in your pregnancy would you like to deliver your baby?" in two different survey questions, with available responses to one question ranging from 6 to 10 months. In the second question, available responses were the correlating GAs in weeks (24-27, $28-31,32-35,36-39$, and 40 weeks or more). Of all survey respondents, $77.6 \%$ of women would like to deliver at 9 months GA, $17.2 \%$ would like to deliver at 10 months, and $5.1 \%$ of women would like to deliver at 8 months or less ( - Table 3). When asked the same question with responses in weeks, $62.9 \%$ of women wanted to deliver at 36 to 9 weeks' gestation and $34.5 \%$ wanted to deliver at 40 weeks or more. Only $2.6 \%$ of patients wanted to deliver earlier than 36 weeks. More non-black women desired delivery at 40 weeks or more, while black women were more likely to desire delivery at 28 to 31 or 32 to 35 weeks $(p=0.005)$. Among the 41 women with a prior preterm birth, the majority (68\%) desired delivery at 36 to 39 weeks' gestation. Only 24\% of women with a history of preterm birth desired delivery at 40 weeks or more compared with $37 \%$ of women without preterm birth $(p=0.08)$. Nearly three quarters of women with current pregnancy complications desired delivery at 36 to 39 weeks or less, compared with $62 \%$ of women with uncomplicated pregnancies ( $p=0.03$ ).

\section{Earliest Gestational Age Perceived to Be Safe for Delivery}

Participants were asked "How far along in pregnancy do you think is the earliest time that it is safe to deliver a baby?" This question was also asked twice on the survey with answers in both months and weeks as previously described. Forty-two percent of women believed that 9 months was the earliest GA at which delivery was safe, while $41.9 \%$ of patients believed that delivery was safe at 8 months GA. More than $14 \%$ of patients thought it was safe to deliver at less than 8 months GA. When asked the same question in weeks, $65.1 \%$ of

Table 3 Gestational ages at which delivery is considered desirable, safe, and full term

\begin{tabular}{|c|c|c|c|c|c|}
\hline Question & \multicolumn{5}{|c|}{ Responses, $n$ (\%) } \\
\hline \multirow[t]{4}{*}{ Desired gestational age at delivery } & $6 \mathrm{mo}$ & $7 \mathrm{mo}$ & $8 \mathrm{mo}$ & $9 \mathrm{mo}$ & $10 \mathrm{mo}$ \\
\hline & $1(0.4)$ & $3(1.3)$ & $8(3.4)$ & $180(77.6)$ & $40(17.2)$ \\
\hline & $24-27$ wk & $28-31$ wk & $32-35$ wk & $36-39$ wk & 40 or more wk \\
\hline & 0 & $1(0.4)$ & $5(2.2)$ & $146(62.9)$ & $80(34.5)$ \\
\hline \multirow{4}{*}{$\begin{array}{l}\text { Earliest gestational age that } \\
\text { delivery is safe }\end{array}$} & $6 \mathrm{mo}$ & $7 \mathrm{mo}$ & $8 \mathrm{mo}$ & $9 \mathrm{mo}$ & $10 \mathrm{mo}$ \\
\hline & $8(3.5)$ & $25(10.9)$ & $96(41.9)$ & $97(42.3)$ & $3(1.3)$ \\
\hline & $24-27$ wk & $28-31 \mathrm{wk}$ & $32-35$ wk & $36-39 w k$ & 40 or more wk \\
\hline & $5(2.2)$ & $11(4.7)$ & $58(24.9)$ & $151(65.1)$ & $7(3.0)$ \\
\hline \multirow[t]{2}{*}{ Gestational age considered full term } & 34 wk & 36 wk & 37 wk & 39 wk & $40 \mathrm{wk}$ \\
\hline & 0 & $27(11.6)$ & $94(40.3)$ & $57(24.5)$ & $55(23.6)$ \\
\hline
\end{tabular}


patients thought that the earliest GA for safe delivery was 36 to 39 weeks. Twenty-five percent believed delivery to be safe at 32 to 35 weeks, and $6.9 \%$ thought that delivery was safe at less than 32 weeks' gestation. There were no significant differences based on sociodemographic characteristics or obstetric history.

\section{Gestational Age for Full-Term Pregnancy}

Survey participants were asked "How far along in pregnancy do you consider a baby to be full term?" with available answers including 34, 36, 37, 39, and 40 weeks. Overall, $40.3 \%$ of patients answered that 37 weeks' gestation was considered full term. More women with complications in their current pregnancy (51\%) believed that full term was reached at 37 weeks as compared with women without complications (34.6\%, $p=0.04$ ) (-Table 3). Respectively, 24.5 and $23.6 \%$ of patients thought that full term was reached at 39 and 40 weeks GA. Twelve percent of all patients considered full term to be reached at 36 weeks GA, while $7.8 \%$ of those patients with current pregnancy complications considered 36 weeks' gestation to be full term. There were no significant differences in responses based on sociodemographic characteristics.

\section{Likelihood of Adverse Neonatal Outcomes}

When asked "If you have a premature baby, what do you think is the chance that your baby will need to be admitted to the intensive care unit (ICU)?" $35.2 \%$ of women considered ICU admission to be very likely, $36.1 \%$ considered it somewhat likely, while $15 \%$ of patients were neutral and $13.9 \%$ considered it to be somewhat or very unlikely (-Table 4). More black women than nonblack women (24 and 7\%, respectively) believed that NICU admission for preterm neonates was somewhat or very unlikely ( $p=0.0003$ ).

Participants were also asked "What do you think is the chance that a premature baby can be breastfed successfully?" Nearly one-third of women believed that successful breastfeeding in a preterm newborn was very likely and $37.8 \%$ believed it was somewhat likely. Twenty-one percent of patients were neutral and only $12.2 \%$ considered it somewhat or very unlikely.

In response to the question "What do you think is the chance that a premature baby will have developmental problems or disabilities?" $39.5 \%$ of participants thought that preterm neonates are very or somewhat likely to have developmental problems, while $34.8 \%$ of patients were neutral and $25.7 \%$ believed it was very or somewhat unlikely.

When asked "What do you think is the chance that a premature baby will leave the hospital at the same time as its mother?" more than one-fourth of women thought that discharge of a preterm neonate at the same time as its mother was very unlikely, and $39.6 \%$ of patients considered it somewhat unlikely.

Similarly, participants were asked "What do you think is the chance that a premature baby may have lifelong health problems?" Thirty-four percent of all patients thought that a neonate born prematurely would be somewhat or very likely to have lifelong health problems, in contrast to the $23.9 \%$ of patients who believed it would be somewhat or very unlikely. Forty-two percent of patients were neutral. Responses did not vary significantly by sociodemographic characteristics or obstetric history.

\section{Experience with Prematurity}

More than half of respondents reported having a friend or family member who had a preterm birth or have experienced it themselves. Among those, $78.7 \%$ of patients reported that the neonate required admission to the NICU. Surprisingly, nearly three quarters of patients surveyed reported that they have never discussed prematurity with a doctor, nurse, or midwife. On the contrary, $69 \%$ of women with prior preterm birth, compared with $18.8 \%$ of women without prior preterm birth reported a discussion with their provider $(p<0.0001)$. Similarly, more women reporting preterm labor in their current pregnancy reported discussing prematurity with their provider $(p=0.03)$. When examined by race, $37 \%$ of black women reported discussing prematurity with their provider, compared with only $20 \%$ of nonblack women $(p=0.003)$. Thirty-two percent of women with high school diplomas and $42 \%$ of women who had completed some college reported discussing prematurity with their providers. Conversely, only 10,11 , and $16 \%$ of women completing less than high school, 4-year college degrees, and graduate degrees, respectively, had discussed prematurity with their providers.

\section{Discussion}

Our results show that most pregnant women find it both safe and desirable to deliver at 9 months or 36 to 39 weeks GA. In

Table 4 Likelihood of various neonatal outcomes

\begin{tabular}{|l|l|l|l|l|l|}
\hline Neonatal outcome & $\begin{array}{l}\text { Very likely, } \\
\boldsymbol{n}(\%)\end{array}$ & $\begin{array}{l}\text { Somewhat } \\
\text { likely, } \boldsymbol{n}(\%)\end{array}$ & $\begin{array}{l}\text { Neutral, } \\
\boldsymbol{n}(\%)\end{array}$ & $\begin{array}{l}\text { Somewhat } \\
\text { unlikely, } \boldsymbol{n}(\%)\end{array}$ & $\begin{array}{l}\text { Very unlikely, } \\
\boldsymbol{n}(\%)\end{array}$ \\
\hline NICU admission & $81(35.2)$ & $83(36.1)$ & $34(14.8)$ & $11(4.8)$ & $21(9.1)$ \\
\hline Successful breastfeeding & $66(28.7)$ & $87(37.8)$ & $49(21.3)$ & $19(8.3)$ & $9(3.9)$ \\
\hline Neurodevelopmental problems & $15(6.5)$ & $76(33.0)$ & $80(34.8)$ & $39(17.0)$ & $20(8.7)$ \\
\hline Discharge at same time as mother & $8(3.5)$ & $25(10.9)$ & $43(18.7)$ & $91(39.6)$ & $63(27.4)$ \\
\hline Lifelong medical problems & $6(2.6)$ & $73(31.7)$ & $96(41.7)$ & $38(16.5)$ & $17(7.4)$ \\
\hline
\end{tabular}

Abbreviation: NICU, neonatal intensive care unit. 
light of the ACOG and NICHD GA definitions, our findings suggest the need for increased provider counseling regarding neonatal risk following preterm and early-term deliveries. ${ }^{4,5}$ Our findings also highlight that certain perceptions varied by race and obstetric history, with more women who were black had a history of prior preterm birth, or with other pregnancy complications desiring delivery at an earlier GA. Interestingly, many patients $(40.3 \%)$ knew that a pregnancy was considered term at 37 weeks and the majority correctly believe that a pregnancy reached full term at 39 to 40 weeks' gestation. Respondents largely believed that adverse neonatal outcomes, such as NICU admission, neurodevelopmental problems, or other lifelong health changes, were very or somewhat likely to occur if a neonate was born prematurely. Finally, pregnant women in our study without baseline risk factors for prematurity overwhelmingly report that they have not spoken to an obstetric provider about prematurity, while those with risk factors for preterm birth reported the contrary.

The variation in responses by race comes as little surprise given the association between black race and preterm birth. ${ }^{8}$ Many studies have identified black women as being at risk of preterm birth, with proposed underlying explanations for this disparity ranging from genetic factors to individual- and community-level socioeconomic factors. ${ }^{9}$ Black women may perceive delivery safer and more acceptable at late preterm and early-term GAs because of an increased collective experience with preterm delivery among themselves, their family, and friends. Given increased exposure to prematurity, they may also perceive adverse neonatal outcomes as being less likely or worrisome if they do occur, as shown in this study by more black than nonblack women believing that NICU admission for a preterm neonate was unlikely.

Obstetric history was also a contributing factor to patients' perceptions of safety and desirability of delivery at different GAs. More patients with a prior preterm birth tended to desire delivery at earlier GAs, which may be due to favorable birth experiences and birth outcomes with their previous preterm deliveries. On the contrary, patients with deliveries at less than 36 weeks or those who had adverse neonatal outcomes may find late preterm or early-term deliveries very desirable compared with their prior preterm birth experiences. Women with current pregnancy complications-who were more likely to desire delivery at 36 to 39 weeks' gestation and to identify 37 weeks as full-term GAhave likely discussed delivery timing with their providers. As such, they may have a different understanding of what is safe and desirable for their pregnancy due to their complications. In this case, the perception of 36 to 39 weeks or 9 months GA as ideal is likely more related to conversations with their providers regarding risks of continued pregnancy versus benefit of delivery rather than misconceptions about the presumed safety of late preterm or early-term birth.

Prior studies that have examined public perceptions of prematurity have identified similar prominent themes, namely, that women desire delivery before 39 weeks' gestation and that misperceptions about the risks of prematurity are prevalent. ${ }^{6,7}$ A major strength of our study is our parti- cipants were pregnant women as compared with prior studies involving postpartum women ${ }^{10}$ and nonpregnant women and men. ${ }^{11}$ Moreover, prior studies have not included pregnant women with prior obstetric complications, which likely influence a pregnant woman's thoughts on what is safe or desirable in her current pregnancy. Prospectively assessing how a pregnant woman feels about the impending outcome of delivery may give a more realistic impression about the factors contributing to decisions made about delivery timing. The anxiety, fears, discomforts, and complications that occur during pregnancy most certainly impact the desire to proceed with delivery prior to 39 weeks, as accounted for in our survey.

Another strength of our study is that all responses were anonymous. This may have reduced response bias as pregnant women may have felt more comfortable answering honestly rather than answering with responses they thought were most desirable by providers. In addition, the study population was diverse in socioeconomic characteristics, including race and insurance status, which may allow responses to be more applicable across broader populations.

One weak point of the study is that obstetric history is based on self-report rather than review of the participants' medical records. As such, results may be subject to misclassification bias as patients may have incorrectly mistaken symptoms or events in their pregnancy as complications that do not meet diagnostic criteria. For example, a woman who delivered her neonate at 37 weeks may inadvertently consider it a preterm rather than early-term delivery. In addition, more than half of the respondents in the survey were patients within a high-risk obstetric practice. While only a minority of women reported having any of the complications listed, most of them likely had some complication in their pregnancy that may have influenced their attitudes and beliefs.

Our results suggest that there is room for improvement in patient education regarding prematurity and appropriate delivery timing. The majority of our respondents reported that they have never had a discussion with an obstetric provider about their risk for preterm birth, with the exception of those with baseline risk factors. Although providers are appropriately counseling at-risk patients when discussing prematurity, a majority of women who deliver preterm neonates are nulliparous or have a history of prior term birth. Innovative educational strategies are needed to better inform all patients about preterm birth. Several models for this type of education have been proposed ranging from individual level to community-based approaches. Armigo has suggested a written teaching tool for all pregnant women and their families focused on fetal development by GA, viability, and survivorship. ${ }^{10}$ Another program used a community-based risk communication approach to promote awareness about preterm birth. ${ }^{11}$ Further research is needed to identify the best strategies to integrate education about healthy delivery timing into routine prenatal care. In addition, our findings suggest a need for targeted interventions designed to influence perspectives on safe delivery timing within the black community where both preterm birth and misconceptions about delivery timing are more common. 
e12 Beliefs about Outcomes by Gestational Age during Pregnancy Baldwin et al.

\section{Significance}

\section{What Is Already Known about the Subject?}

Previous studies in the nonpregnant and postpartum populations demonstrate widespread misconceptions about definitions of a term gestation and the risks of delivery at varied GAs.

\section{What Does This Study Add?}

The current study examines perceptions about what GA defines a term pregnancy and neonatal risks associated with delivery at varied GAs in women who are currently pregnant. Additionally, the current study examines differences in responses based on risk factors for preterm birth including race, obstetrical history and pregnancy comorbidities.

\section{Conflict of Interest}

None.

\section{References}

1 Tita AT, Landon MB, Spong CY, et al; Eunice Kennedy Shriver NICHD Maternal-Fetal Medicine Units Network. Timing of elective repeat cesarean delivery at term and neonatal outcomes. N Engl J Med 2009;360(02):111-120
2 Clark SL, Miller DD, Belfort MA, Dildy GA, Frye DK, Meyers JA. Neonatal and maternal outcomes associated with elective term delivery. Am J Obstet Gynecol 2009;200(02):156.e1-156.e4

3 Cheng YW, Nicholson JM, Nakagawa S, Bruckner TA, Washington $\mathrm{AE}$, Caughey AB. Perinatal outcomes in low-risk term pregnancies: do they differ by week of gestation? Am J Obstet Gynecol 2008;199(04):370.e1-370.e7

4 Spong CY. Defining "term" pregnancy: recommendations from the Defining “Term" Pregnancy Workgroup. JAMA 2013;309(23): 2445-2446

5 ACOG Committee Opinion No 579: definition of term pregnancy. Obstet Gynecol 2013;122(05):1139-1140

6 Goldenberg RL, McClure EM, Bhattacharya A, Groat TD, Stahl PJ. Women's perceptions regarding the safety of births at various gestational ages. Obstet Gynecol 2009;114(06):1254-1258

7 Massett HA, Greenup M, Ryan CE, Staples DA, Green NS, Maibach EW. Public perceptions about prematurity: a national survey. Am J Prev Med 2003;24(02):120-127

8 Culhane JF, Goldenberg RL. Racial disparities in preterm birth. Semin Perinatol 2011;35(04):234-239

9 Bryant AS, Worjoloh A, Caughey AB, Washington AE. Racial/ethnic disparities in obstetric outcomes and care: prevalence and determinants. Am J Obstet Gynecol 2010;202(04):335-343

10 Armigo C. Prenatal education regarding gestational development, viability, and survivorship: looking to our obstetric colleagues for change. Adv Neonatal Care 2008;8(03):185-189

11 Wright K, Jones L, Hogan V. A roadmap for authentic community/ academic engagement for developing effective community preterm birth education. Ethn Dis 2010;20(01, Suppl 2):S2-S77, 82 